\title{
Skewer: a fast and accurate adapter trimmer for next-generation sequencing paired-end reads
}

\author{
Hongshan Jiang ${ }^{1 *}$, Rong Lei ${ }^{1}$, Shou-Wei Ding ${ }^{2}$ and Shuifang Zhu ${ }^{1}$
}

\begin{abstract}
Background: Adapter trimming is a prerequisite step for analyzing next-generation sequencing (NGS) data when the reads are longer than the target DNA/RNA fragments. Although typically used in small RNA sequencing, adapter trimming is also used widely in other applications, such as genome DNA sequencing and transcriptome RNA/cDNA sequencing, where fragments shorter than a read are sometimes obtained because of the limitations of NGS protocols. For the newly emerged Nextera long mate-pair (LMP) protocol, junction adapters are located in the middle of all properly constructed fragments; hence, adapter trimming is essential to gain the correct paired reads. However, our investigations have shown that few adapter trimming tools meet both efficiency and accuracy requirements simultaneously. The performances of these tools can be even worse for paired-end and/or mate-pair sequencing.
\end{abstract}

Results: To improve the efficiency of adapter trimming, we devised a novel algorithm, the bit-masked k-difference matching algorithm, which has $O(k n)$ expected time with $O(m)$ space, where $k$ is the maximum number of differences allowed, $n$ is the read length, and $m$ is the adapter length. This algorithm makes it possible to fully enumerate all candidates that meet a specified threshold, e.g. error ratio, within a short period of time. To improve the accuracy of this algorithm, we designed a simple and easy-to-explain statistical scoring scheme to evaluate candidates in the pattern matching step. We also devised scoring schemes to fully exploit the paired-end/mate-pair information when it is applicable. All these features have been implemented in an industry-standard tool named Skewer (https:// sourceforge.net/projects/skewer). Experiments on simulated data, real data of small RNA sequencing, paired-end RNA sequencing, and Nextera LMP sequencing showed that Skewer outperforms all other similar tools that have the same utility. Further, Skewer is considerably faster than other tools that have comparative accuracies; namely, one times faster for single-end sequencing, more than 12 times faster for paired-end sequencing, and 49\% faster for LMP sequencing.

Conclusions: Skewer achieved as yet unmatched accuracies for adapter trimming with low time bound.

Keywords: Next generation sequencing, Adapter trimming, Approximate string matching, Local sequence alignment, Barcode demultiplexing

\section{Background}

Adapter sequences are short oligonucleotides used to be ligated to the ends of DNA fragments of interest, so that they can be combined with primers for amplification. When the sequencing read length is greater than that of the target DNA, the adapter sequence is read out, sometimes partially, next to the unknown target DNA sequence. To recover the target DNA sequence, it is essential to identify the adapter sequence and trim it.

\footnotetext{
*Correspondence: hongshan.jiang@gmail.com

${ }^{1}$ Institute of Plant Quarantine Research, Chinese Academy of Inspection and Quarantine, Huixinli 241, Beijing, 100029 China

Full list of author information is available at the end of the article
}

Adapter trimming was first used in small RNA (sRNA) sequencing, where typical lengths of the target fragments range from 18 nucleotides (nt) to $30 \mathrm{nt}$, while the typical read length is $36 \mathrm{nt}$. Another important application for adapter trimming is DNase-Seq, which is a highresolution technique used to profile hypersensitive sites that are frequently bound by transcription factors. Recent studies showed that the sequencing of short reads (50-100 base pairs (bp)) gives better results [1].

For all the next-generation sequencing (NGS) applications including chromosomal DNA sequencing or complementary DNA (cDNA) sequencing, double-stranded DNA is first fragmented using nebulization or ultrasonics to obtain lengths of several hundreds bp. The ends of 
the fragments are then repaired and ligated with adapters. After purification, the adapter-ligated fragments are either poured on slides/chips as water-in-oils or attached to flow-cells for cluster generation. After several cycles of emulsion-PCR or bridge-PCR, the amplified templates are ready for sequencing. Fragmentation is a stochastic process that is influenced by the varied force field and thermodynamic stability of different parts of the sequence; e.g. parts with different GC content. To improve the enrichment rate and to cover the target genome or transcriptome more evenly, the sample preparation protocol usually requires fragments to be enriched in a specified length range. Even when longer average fragment lengths are chosen, the inherent nature of fragment size selection cannot always prevent short fragments and primerdimmers from going on to the next stage. Therefore, there are nearly always reads that need to be trimmed. For the newly emerged Nextera long mate-pair (LMP) protocol, junction adapters that connect paired target fragments exist in all properly constructed fragments; thus, adapter trimming is essential to gain correct paired reads.

Adapter trimming is different from contaminant removal and is usually associated with NGS protocols where adapters are synthesized and specified by the reagent vendors. Given a known adapter pattern and a read sequence, adapter trimming is usually modeled as a semi-global sequence alignment, also called end-space free alignment, where any space at the end of or beginning of the alignment does not incur penalties.

Semi-global sequence alignment can be performed using the Smith-Waterman algorithm [2] with minor revisions of the boundary condition as implemented in Cutadapt [3], which has a time complexity of $O(m n)$, where $m$ is the pattern length, and $n$ is the sequence length. A trickier solution, i.e. Ukkonen's algorithm for $k$ difference matching [4], has an expected time of $O(k n)$, where $k<m$ is the maximum number of differences allowed. Further improvements in Ukkonen's algorithm by bitwise parallelism were proposed by Myer [5] and implemented in Btrim [6], which has a time complexity of $O(m n / w)$, where $w$ is the word length of the computer; e.g. $w$ equals 64 for a 64-bit machine. In practice, Myer's bit-vector dynamic programming algorithm is the fastest $k$-difference matching algorithm currently available. However, Myer's algorithm is more appropriate for searching patterns in text and is difficult to adapt to deal with sequencing quality values in NGS data.

Other popular adapter trimmers are available; e.g. Fastx_clipper in the FastX-Toolkit (http://hannonlab.cshl. edu/fastx_toolkit/), SeqTrim (https://github.com/dariogf/ SeqtrimNext) [7], TagCleaner [8], and EA-Tools (http:// code.google.com/p/ea-utils/). Recently published trimmers are: SeqPrep (https://github.com/jstjohn/SeqPrep) which focuses on paired-end (PE) reads; Flexbar [9], a flexible barcode demultiplexer that uses the NeedlemanWunsch algorithm [10] for pair-wise global sequence alignments, which has the same time complexity as that of Smith-Waterman algorithm; Trimmomatic (http://www. usadellab.org/cms/index.php?page=trimmomatic), which is a part of an integrated tool RobiNA [11]; Scythe (https://github.com/vsbuffalo/scythe), which uses a Naive Bayesian approach to classify contaminants in reads; TrimGalore (http://www.bioinformatics.babraham.ac.uk/ projects/trim_galore/), which internally invokes Cutadapt with an extension to handle PE reads; AdapterRemoval [12], which is carefully tuned for trimming adapters from both single-end (SE) or PE reads; AlienTrimmer [13], which is based on $k$-mer decomposition for contaminant detection; and NextClip [14], which is dedicated to trimming adapters within Nextera LMP reads.

For sRNA sequencing data, a simple script can handle the adapter trimming task with acceptable accuracy and speed. However, trimming adapter sequences from genome sequencing data requires tools that are much more efficient, because the volume of data is much larger. Some very fast tools such as Btrim and Trimmomatic tend to over-simplify the underlying model. Btrim neglects the quality values associated with base calls for adapter trimming although it does use quality values for quality trimming [6]. Trimmomatic adopts a hashbased search followed by a simple score-based search, both of which neglect insertions and deletions for adapter matching (http://www.usadellab.org/cms/index.php? page $=$ trimmomatic). To gain sufficient accuracy, most of the adaptor trimming algorithms use conventional pairwise alignment algorithms such as the Smith-Waterman or Needleman-Wunsch algorithms; however, these alignment algorithms are inefficient for adapter trimming compared with more sophisticated algorithms [5].

In a typical application of adapter trimming, e.g. for sRNA sequencing, usually only a short prefix of the fulllength adapter is scanned to reduce run time. However, this strategy may increase the possibility of random hits and cause biases in the trimmed sequences. Even when the specified pattern is of sufficient length, for reads where the 3 ' end overlaps with a short prefix of the adaptor pattern, it is hard to judge whether the overlap is from finding the adapter sequence or from sequence homology. On the one hand, over-trimming of a bona fide part of the sequence causes loss of information; on the other hand, leaving an adapter untrimmed causes noise in the downstream analysis.

In this paper, we propose a bit-masked $k$-difference matching dynamic programming algorithm with $O(k n)$ expected time and $O(m)$ space in which the information within the adapter sequences is transferred into bits. We developed a carefully designed statistical scheme that 
incorporates quality values (and PE information when applicable), and implemented an industry-standard Linux program called Skewer to address the trimming problem accurately and efficiently.

\section{Results and discussions}

\section{Features}

The algorithm and statistical scoring schemes are implemented as a Linux program Skewer using C++. A comparison of the main features of Skewer with those of existing mainstream adapter trimmers are presented in Table 1.

\section{Experiment environment}

The server that was used for the experiments had $4 \times 8$ core Intel $^{\circ} 2.67 \mathrm{GHz}$ CPUs, $1 \mathrm{~T}$ memory, and RAID with bandwidths of $266 \mathrm{MB} / \mathrm{s}$ and $262 \mathrm{MB} / \mathrm{s}$ for reading and writing respectively. The operating system (OS) was the Red Hat Enterprise Linux Server release 6.3.

\section{Experiments on simulated data General information}

We simulated 10 million $100 \mathrm{bp}+100 \mathrm{bp}$ PE Solexa reads from the Arabidopsis thaliana genome using ART, a NGS read simulator [15], with some revision on the source codes for simulating adapter-contaminated reads (http://sourceforge.net/projects/skewer/files/Simulator/). The trained profile was from the real sequencing data of $A$. thaliana where about $36 \%$ of the reads were contaminated with adapters. We compared Skewer with mainstream adapter trimmers that can handle PE reads as well as four representative adapter trimmers that can handle only SE reads.

To assess trimming quality, we defined the following metrics: FP (false positive) as the number of reads that were over-trimmed, either for trimming non-contaminant reads (false trimming), noted as $F P f t$, or for overtrimming contaminant reads, noted as FP_ot; FN (false negative) as the number of reads that were undertrimmed, either for not trimming contaminant reads (false retaining), noted as $F N_{f} f r$, or under-trimming contaminant reads, noted as $F N \_u t$; and TN (true negative) as the number of untrimmed non-contaminant reads.

From these numbers, we defined the positive predictive value (PPV) as the ratio of the number of correctly trimmed reads to the number of trimmed reads; sensitivity (Sen) as the ratio of the number of correctly trimmed reads to the number of contaminant reads; and specificity (Spec) as the ratio of the number of untrimmed noncontaminant reads to the number of non-contaminant reads as follows:

$$
\begin{aligned}
P P V & =T P /\left(T P+F P \_t+F P \_o t+F N_{\_} u t\right) \\
\text { Sen } & =T P /\left(T P+F N \_f r+F N_{-} u t+F P \_o t\right) \\
\text { Spec } & =T N /\left(T N+F P \_f t\right)
\end{aligned}
$$

\begin{tabular}{|c|c|c|c|c|c|c|c|c|c|c|c|c|}
\hline \multirow[b]{2}{*}{ Method } & \multicolumn{6}{|c|}{ Adapter trimming } & \multicolumn{2}{|c|}{ Quality control } & \multicolumn{4}{|c|}{ Other } \\
\hline & $5^{\prime}$ & $3^{\prime}$ & SE & $\mathrm{PE}$ & LMP & Multi & Ns & $Q$ & Barcode & Merge & gzip Files & MT \\
\hline FastX & $x$ & $\bigcirc$ & O & $x$ & $x$ & $x$ & $\bigcirc$ & $\times$ & $\bigcirc$ & $x$ & $x$ & $x$ \\
\hline SeqTrim & $x$ & $\bigcirc$ & $\bigcirc$ & $\times$ & $x$ & $\bigcirc$ & $\bigcirc$ & $\bigcirc$ & $x$ & $x$ & $\bigcirc$ & $\bigcirc$ \\
\hline TagCleaner & $\bigcirc$ & $\bigcirc$ & $\bigcirc$ & $\times$ & $x$ & $x$ & $x$ & $x$ & $x$ & $x$ & $x$ & $x$ \\
\hline EA-Tools & $\times$ & $\bigcirc$ & $\bigcirc$ & $\bigcirc$ & $\times$ & $x$ & $\bigcirc$ & $\bigcirc$ & 0 & $x$ & $\bigcirc$ & $x$ \\
\hline Cutadapt & $\bigcirc$ & $\bigcirc$ & $\bigcirc$ & $\bigcirc$ & $x$ & $\bigcirc$ & $x$ & $\bigcirc$ & $x$ & $x$ & $\bigcirc$ & $x$ \\
\hline TrimGalore & $\times$ & $\bigcirc$ & $\bigcirc$ & $\bigcirc$ & $x$ & $x$ & $\times$ & $\bigcirc$ & $x$ & $x$ & $\bigcirc$ & $x$ \\
\hline SeqPrep & $\times$ & $\bigcirc$ & $\times$ & $\bigcirc$ & $\times$ & $x$ & $\times$ & $\times$ & $x$ & $\bigcirc$ & $\times$ & $x$ \\
\hline Btrim & $\bigcirc$ & $\bigcirc$ & 0 & 0 & $x$ & $x$ & $x$ & $\bigcirc$ & 0 & $x$ & $x$ & $x$ \\
\hline Scythe & $\times$ & $\bigcirc$ & $\bigcirc$ & $\times$ & $x$ & $x$ & $\times$ & $\times$ & $x$ & $x$ & $\bigcirc$ & $x$ \\
\hline Flexbar & 0 & $\bigcirc$ & 0 & 0 & $x$ & 0 & $\bigcirc$ & $\bigcirc$ & 0 & $x$ & $\bigcirc$ & 0 \\
\hline Trimmomatic & $x$ & $\bigcirc$ & 0 & $\bigcirc$ & $x$ & $\bigcirc$ & $x$ & $\bigcirc$ & $x$ & $x$ & $\bigcirc$ & 0 \\
\hline AdapterRemoval & $\bigcirc$ & $\bigcirc$ & $\bigcirc$ & $\bigcirc$ & $x$ & $x$ & $\bigcirc$ & $\bigcirc$ & $x$ & $\bigcirc$ & $x$ & $x$ \\
\hline AlienTrimmer & $\bigcirc$ & $\bigcirc$ & $\bigcirc$ & $\bigcirc$ & $x$ & $\bigcirc$ & $x$ & $\bigcirc$ & $x$ & $x$ & $x$ & $x$ \\
\hline NextClip & $x$ & $x$ & $x$ & $\times$ & $\bigcirc$ & $x$ & $x$ & $x$ & $x$ & $x$ & $x$ & $x$ \\
\hline Skewer & $\bigcirc$ & $\bigcirc$ & $\bigcirc$ & $\bigcirc$ & $\bigcirc$ & $\bigcirc$ & $\bigcirc$ & $\bigcirc$ & $\bigcirc$ & $\times$ & $\bigcirc$ & 0 \\
\hline
\end{tabular}

Table 1 Main features of various adapter trimmers

For each method, the table shows if it is able to: i) identify adapters in the $5^{\prime}$ end of reads, ii) identify adapters in the $3^{\prime}$ end of reads, iii) process single-end (SE) reads, iv) process paired-end (PE) reads, v) process Nextera long mate-pair (LMP) reads, vi) search for multiple different adapters (Multi), vii) trim subsequences of multiple degenerative characters (Ns), viii) trim low-quality nucleotides (Q), ix) separate multiplexed reads based on barcodes, $x$ ) merge overlapped pairs into longer single-end

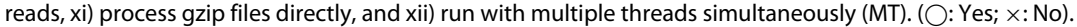


Finally, we defined the Matthew's correlation coefficient $(\mathrm{mCC})$, which is a quality measure for pattern recognition, as:

$$
m C C=\frac{T P \cdot T N-F P \cdot F N}{\sqrt{(T P+F P)(T P+F N)(T N+F P)(T N+F N)}}
$$

\section{Primary result}

Each method was run with its default parameters, except that the minimum output fragment length and the thread number (if applied) were set to 1 .

The results obtained from these runs are listed in Table 2 and details are available in Table S1 of Additional file 1. Fast X, an earlier and widely adopted NGS adapter trimmer, had a relatively low $m C C(0.6683)$ and a low processing speed $(0.92 \mathrm{Mbp} / \mathrm{s})$; SeqTrim had a similar overall performance as Fast $X$ (0.6618), but it had the slowest processing speed $(0.03 \mathrm{Mbp} / \mathrm{s})$ of all the trimmers tested despite its extensive logging utility; TagCleaner was clearly the most conservative of the trimmers $(F P=0)$, but it had the lowest sensitivity (45.50\%) and was notably slower than FastX (58.7\% of the speed); EA-Tools had the highest sensitivity (99.72\%) for processing SE reads and was orders of magnitude faster $(13 X \sim 400 X)$ than the slow trimmers; Cutadapt, the most widely accepted adapter trimmer, exhibited a good compromise between sensitivity and specificity (96.27\% vs. $96.93 \%)$, and had the highest $m C C(0.9286)$ among the existing tools for processing SE reads; TrimGalore, a wrapper for Cutadapt, had a performance that was equivalent to $E A$-tools with default settings, but it was considerably slower than $E A$ tools $(28.2 \% \sim 31.6 \%$ of the speed); SeqPrep, a dedicated $\mathrm{PE}$ reads adapter trimmer and merger, had the highest $m C C$ (0.9975) among the existing tools for processing PE reads, but it was slow $(0.64 \mathrm{Mbp} / \mathrm{s})$; Btrim had the highest speed $(23.63 \mathrm{Mbp} / \mathrm{s})$ for adapter trimming, but it had low sensitivity (53.44\%); Scythe had an $m C C$ similar to that of Cutadapt for SE reads adapter trimming, but was more conservative; Flexbar had slightly lower metrics and about 20\% lower processing speed than TrimGalore; Trimmomatic was among the most conservative ones, but it had

Table 2 Performance of adapter trimmers on 2Gbp simulated data

\begin{tabular}{|c|c|c|c|c|c|c|c|}
\hline \multicolumn{2}{|c|}{ Method (Single End/Paired End) } & \multirow{2}{*}{$\begin{array}{r}\text { Speed (Mbp/s) } \\
0.92\end{array}$} & \multirow{2}{*}{$\begin{array}{r}\text { Memory (Mb) } \\
13.8 \\
\end{array}$} & \multirow{2}{*}{$\begin{array}{r}\text { PPV (\%) } \\
68.90\end{array}$} & \multirow{2}{*}{$\begin{array}{r}\text { Sen. (\%) } \\
90.84\end{array}$} & \multirow{2}{*}{$\begin{array}{r}\text { Spec. (\%) } \\
77.97\end{array}$} & \multirow{2}{*}{$\begin{array}{r}\mathrm{mCC} \\
0.6683\end{array}$} \\
\hline FastX & SE & & & & & & \\
\hline SeqTrim & SE & 0.03 & 115.7 & 67.07 & 85.27 & 81.24 & 0.6618 \\
\hline TagCleaner & SE & 0.54 & 37.6 & 100.0 & 45.50 & 100.0 & 0.5898 \\
\hline \multirow{2}{*}{ EA-Tools } & SE & 12.04 & 17.7 & 59.24 & 99.72 & 61.32 & 0.6010 \\
\hline & PE & 11.54 & 30.0 & 59.16 & 99.43 & 61.36 & 0.5983 \\
\hline \multirow{2}{*}{ Cutadapt } & $\mathrm{SE}$ & 4.36 & 34.5 & 94.55 & 96.27 & 96.93 & 0.9286 \\
\hline & PE & 3.44 & 42.8 & 94.55 & 96.00 & 96.93 & 0.9266 \\
\hline \multirow{2}{*}{ TrimGalore } & SE & 3.81 & 19.4 & 59.24 & 99.72 & 61.32 & 0.6010 \\
\hline & PE & 3.26 & 19.6 & 59.16 & 99.44 & 61.36 & 0.5984 \\
\hline SeqPrep & PE & 0.64 & 22.0 & 99.84 & 99.82 & 99.92 & 0.9975 \\
\hline \multirow{2}{*}{ Btrim } & SE & 23.63 & 11.2 & 99.96 & 53.44 & 100.0 & 0.6503 \\
\hline & PE & 5.79 & 15.3 & 99.89 & 53.30 & 100.0 & 0.6490 \\
\hline Scythe & SE & 3.15 & 11.2 & 99.56 & 90.86 & 99.92 & 0.9283 \\
\hline \multirow{2}{*}{ Flexbar } & SE & 2.82 & 9.5 & 57.90 & 99.12 & 59.48 & 0.5814 \\
\hline & PE & 2.70 & 9.7 & 57.77 & 99.09 & 59.29 & 0.5795 \\
\hline \multirow{2}{*}{ Trimmomatic } & $\mathrm{SE}$ & 16.73 & 2593.0 & 99.99 & 72.31 & 100.0 & 0.7907 \\
\hline & PE & 16.40 & 2292.0 & 100.0 & 71.54 & 100.0 & 0.7850 \\
\hline \multirow{2}{*}{ AdapterRemoval } & SE & 1.67 & 6.3 & 75.09 & 97.74 & 81.89 & 0.7675 \\
\hline & PE & 0.73 & 8.3 & 99.93 & 94.47 & 99.97 & 0.9566 \\
\hline \multirow{2}{*}{ AlienTrimmer } & $\mathrm{SE}$ & 1.64 & 2319.9 & 85.62 & 57.11 & 99.96 & 0.6769 \\
\hline & PE & 1.61 & 2248.9 & 83.71 & 55.67 & 99.95 & 0.6659 \\
\hline \multirow{2}{*}{ Skewer } & $\mathrm{SE}$ & 8.79 & 13.6 & 94.56 & 96.32 & 96.93 & 0.9291 \\
\hline & $P E$ & 8.88 & 22.2 & 100.0 & 99.86 & 100.0 & 0.9989 \\
\hline
\end{tabular}

Methods that process only single-end (SE) or paired-end ( $\mathrm{PE}$ ) reads are indicated. 
an acceptable sensitivity (72.31\%) and a relatively high speed $(16.73 \mathrm{Mbp} / \mathrm{s})$; AlienTrimmer had similar metrics to Btrim, but was much slower $(1.64 \mathrm{Mbp} / \mathrm{s})$; and AdapterRemoval had a similar overall performance as SeqPrep for PE reads processing, but unlike SeqPrep it can also handle SE reads.

The results of the runs listed in Table 2 show that Skewer outperformed all the mainstream tools in terms of $m C C$ for both SE and PE trimming (0.9291 and 0.9989 respectively), although Skewer was only marginally better than Cutadapt and Scythe in SE trimming. Furthermore, Skewer was substantially faster (one times faster for SE and more than 12 times faster for PE trimming) than the tools that had comparative performances.

Trimmomatic and AlienTrimmer both used above $2 G$ bytes peak memory. Most of the other trimmers used less than $50 M$ bytes memory except SeqTrim, which used $115.7 M$ bytes. Although Skewer did not have the least memory usage, its memory consumption (less than $35 \mathrm{M}$ bytes, see Table S2 of Additional file 1 for details) was far from a bottleneck on a 64-bit computer. In fact, Skewer uses additional memory to facilitate the processing of IUPAC (International Union of Pure and Applied Chemistry) characters and for parallel computing.

\section{Scalability for parallel computing}

We ran various adapter trimmers that support multithreading to compare their scalability under a parallel computing environment. SeqTrim was excluded because it is too slow to gain comparative speed even with tens of threads. In addition, Cutadapt, AdapterRemoval, and other tools were not included because they currently lack a multi-threading function. In the eight threads case, for both the uncompressed and compressed inputs, Skewer achieved the highest speedup among the adapter trimmers tested ( 7.87 for uncompressed input, and 4.54 for compressed input) (see Table S2 of Additional file 1 for details).

\section{Receiver operating characteristic (ROC) curves}

ROC curves for various adapter trimmers under different stringencies were plotted and are shown in Figure 1 and Figure 2 (see Table S3 of Additional file 1 for details).

In high stringency (left) regions, both Trimmomatic and Cutadapt performed well in that they had low FPRs (false positive rates) and high TPRs (true positive rates); however, as the stringency decreases, both their performance degrade gradually (Figure 1). A similar trend was seen for TrimGalore where the ROC curve shifted to the upperright region. This implies that these trimmers greedily picked up the first candidate that met the stringency rather than select the optimal one. The ROC curve for AlienTrimmer was similar to the above ones, but with a worse performance. Fast $X$ may adopt some optimization technique, however, its performance was worse than those of Trimmomatic and Cutadapt within all the stringency range. Other adapter trimmers showed advantages on a specific metric; e.g. AdapterRemoval, Flexbar, and EATools were the most sensitive, while TagCleaner, Btrim, and Scythe were the most conservative. SeqTrim appeared only as a dot in the ROC curves plot, because it does not provide a stringency threshold. Skewer outperformed the other adapter trimmers in that it had the least FPR to gain a specific TPR, when TPR $>95 \%$.

ROC curves for the adapter trimmers that are aware of PE information were plotted and are shown in Figure 2. Other trimmers that can process PE reads have worse ROC curves than corresponding ROC curves for processing SE reads since the second reads usually have lower sequencing qualities. From Figure 2, we can see that Skewer had a nearly perfect ROC curve close to the upperleft corner. For example, it achieved a TPR of $99.951 \%$ with a FPR of $0.001 \%$.

\section{Experiments on real data sRNA sequencing data for Caenorhabditis elegans}

A recently published real sRNA data set (short read archive [SRA:SRR014966]) [16], which includes 14,251,981 reads of small non-coding RNA (ncRNA) from C. elegans, was used to evaluate the adapter trimmers. Because it is hard to recover all the underlying sRNA fragments for sequencing, we aligned the trimmed reads to the reference genome and used delta of the number of uniquely aligned reads relative to the number of uniquely aligned raw reads, noted as TT (true trimming), as a substitute for true positive. We also used delta of number of non-uniquely aligned reads relative to the number of nonuniquely aligned raw reads, noted as FT (false trimming), as a substitute for false positive. The rationale was that correct-trimming tends to change unaligned fragments to uniquely aligned fragments (true positive), while overtrimming tends to change uniquely aligned fragments to non-uniquely aligned fragments (false positive). Note that these metrics tolerate tiny mistakes that can be rescued by the alignment software and are useful for practical evaluation.

To evaluate the performances under various trimming stringencies, all the tools were used to trim adapter sequences from the C. elegans data set using various trimming stringency. Next the processed reads were aligned to the C. elegans genome [17] (version 10) using Bowtie2 [18] (version 2.1.0). We then used the above metrics for final plotting, with higher $F T$ representing lower stringency.

The results are presented in Table S4 and Table S5 of Additional file 2, and illustrated in Figure 3. AdapterRemoval and Flexbar exhibited similar performance curves, while AdapterRemoval was slightly better than Flexbar within all the tested stringency range; and TrimGalore 


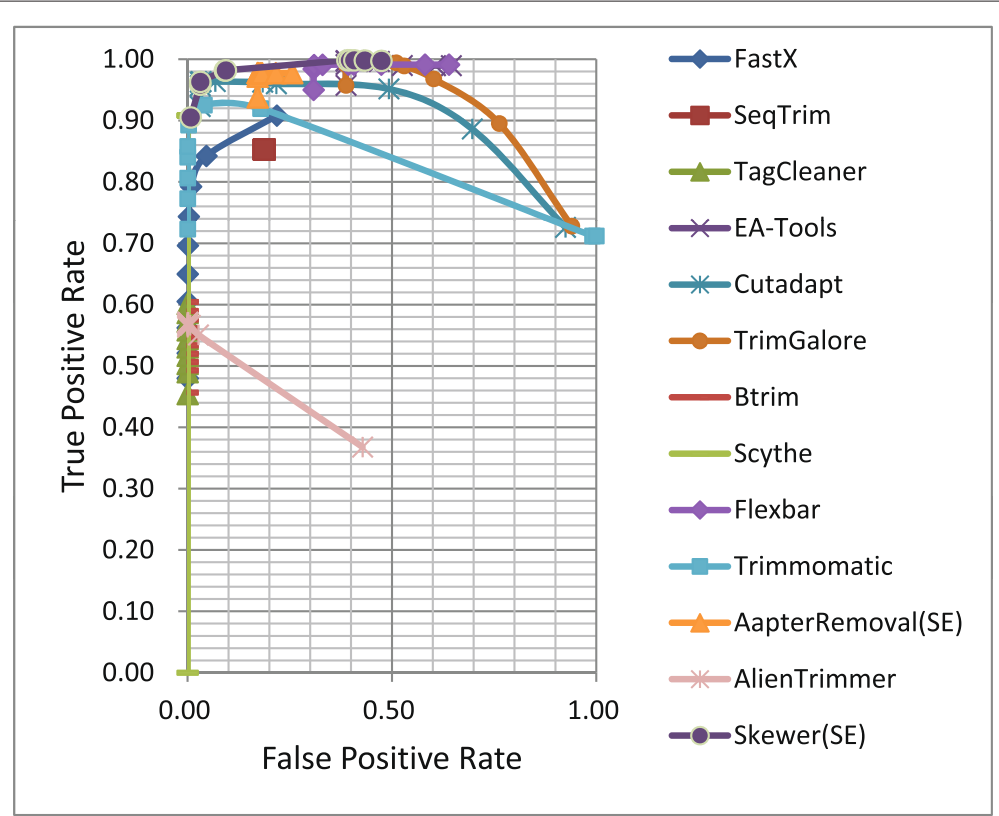

Figure 1 ROC curves of various adapter trimmers for processing single-end reads of simulated data. ROC: receiver operating characteristic.

and Cutadapt had similar curves, while TrimGalore was slightly better than Cutadapt at all the stringencies. Under high stringency, EA-Tools, Skewer, and TrimGalore shared the first rank in terms of low FT and high TT; Trimmomatic, AdapterRemoval, and Schythe were ranked second under middle stringency, middle low stringency, and low stringency respectively; and Skewer ranked first at all the stringencies.
Paired-end RNA sequencing data for Drosophila simulans

A real RNA-Seq data set with 27,005,344 pairs of 101 bp reads (short read archive [SRA:SRR330569]) from the gonads and carcasses of $D$. simulans was used to compare the performances of the adapter trimmers in trimming artificial contaminants from PE reads.

For the evaluation, we first used each of the tools that can deal with PE reads with default setting to trim

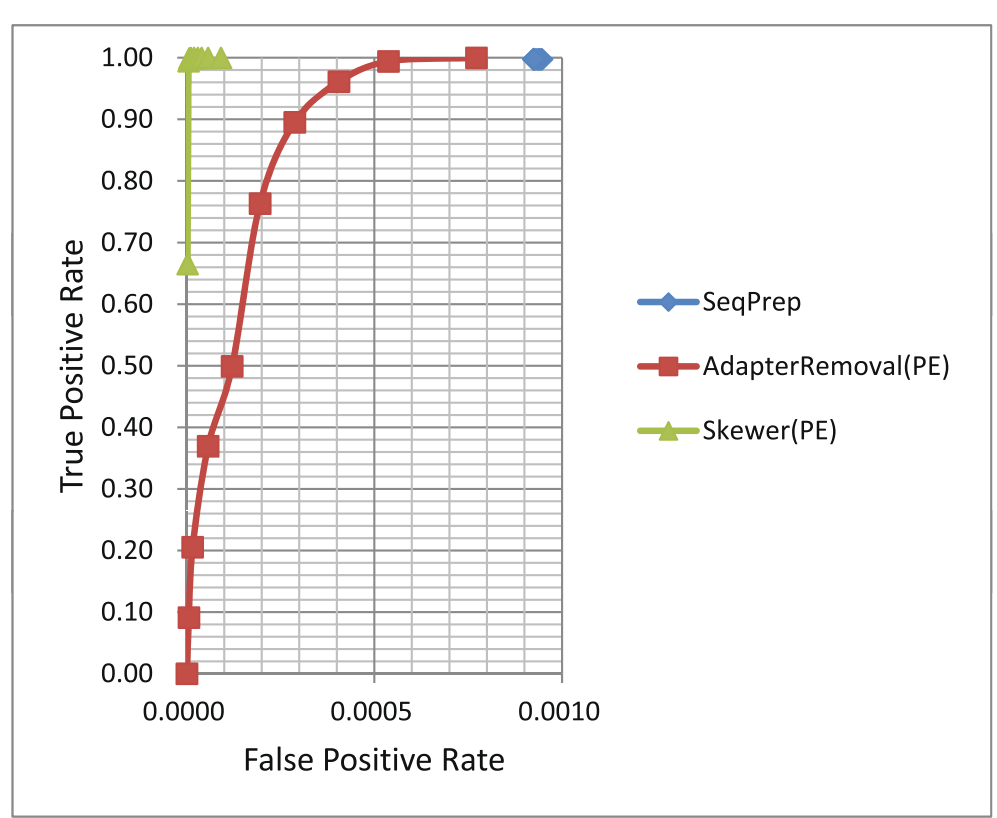

Figure 2 ROC curves of various adapter trimmers for processing paired-end reads of simulated data. ROC: receiver operating characteristic. 


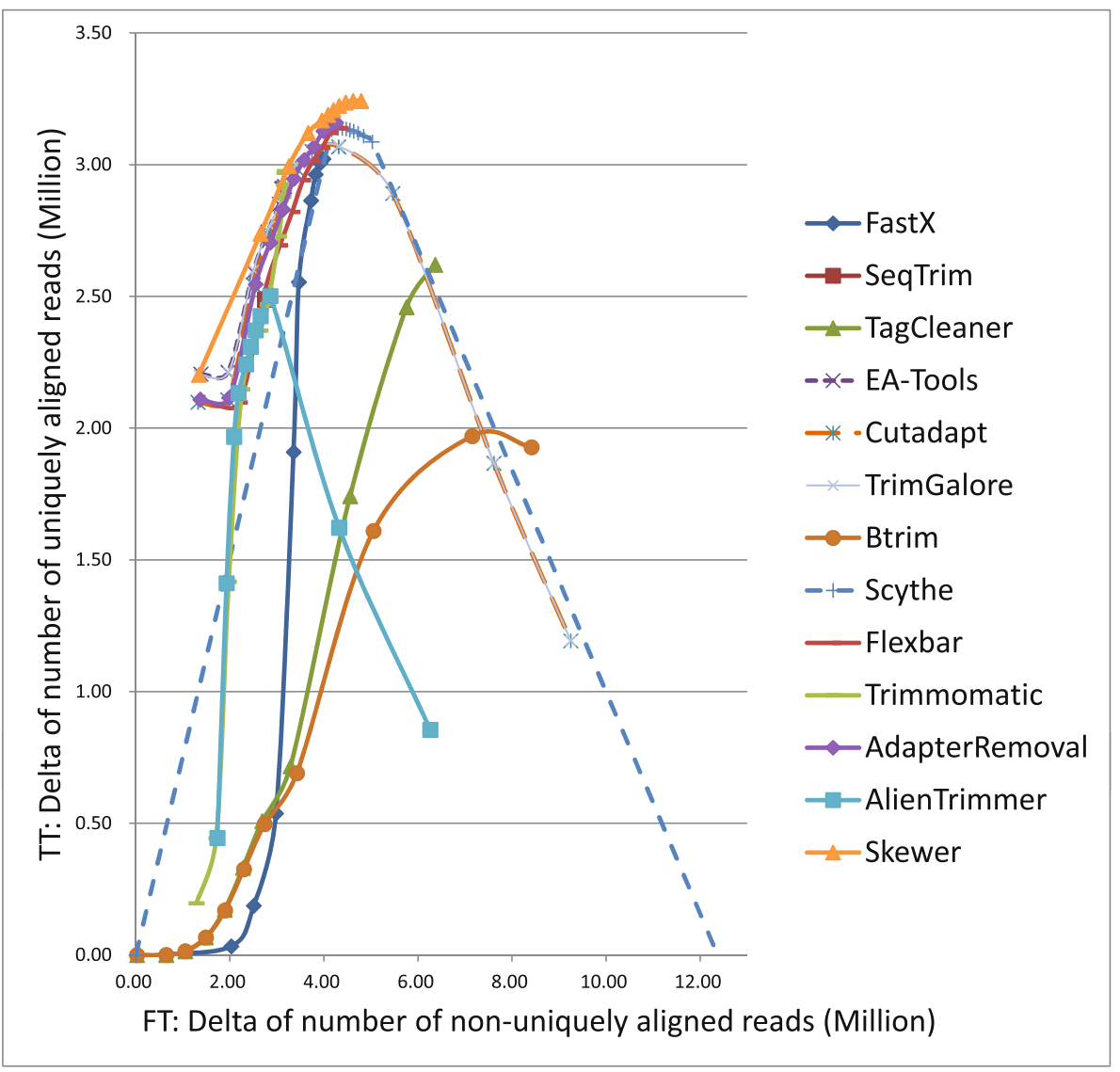

Figure 3 Performance of various adapter trimmers on real small RNA data [SRA:SRR014966].

adapters from the reads, with the exception that the minimum output fragment length was set to 20 and quality trimming was inhibited. We then used TopHat $[19,20]$ (version 2.0.10) to align the processed reads to the reference genome of $D$. simulans [21] (dsim revision 1.4). Finally the number of uniquely and concordantly aligned pairs was used as the performance metrics.

The results are presented in Table S6 of Additional file 3 and illustrated in Figure 4. Skewer outperformed the other adapter trimmers in terms of the number of uniquely and concordantly aligned pairs of the trimmed PE reads. Trimmomatic and AdapterRemoval, both of which performed well in processing the sRNA data, performed poorly in processing the long PE data. This finding implies that these tools may be tuned specifically for trimming adapters from sRNA data. Similarly, Btrim also performed less well with the PE data in this experiment. After investigating the processed data, we found that Btrim could recognize only the occurrence of the whole adapter sequence with a limited tolerance for insertions and deletions. It should be noted that all quality trimming was inhibited from these experiments to compare the adapter trimming performance alone. However, in real applications, quality trimming, which is outside of the scope of this paper, has been reported to improve the mapping rate and facilitate downstream data analysis [22].

\section{Nextera long mate pair (LMP) data for Arabidopsis thaliana}

A 5-kb insert size Nextera LMP library of $A$. thaliana Col-0 with 6,602,426 pairs of 251bp reads (European Nucleotide Archive [ENA:ERA264981]) and a 400-bp insert size Illumina HiSeq PE library of the same species with 17,341,797 pairs of 100 bp reads [ENA:SRR519624] were sequenced previously and used to demonstrate the utility of NextClip [14], a dedicated tool for trimming adapters from Nextera LMP libraries.

To compare Skewer with NextClip, we followed a validation procedure similar to the one described for NextClip [14]. Briefly, the LMP library was first trimmed using the adaptor trimmer. Then the trimmed LMP reads and the PE reads were de novo assembled using ABySS [23]. 


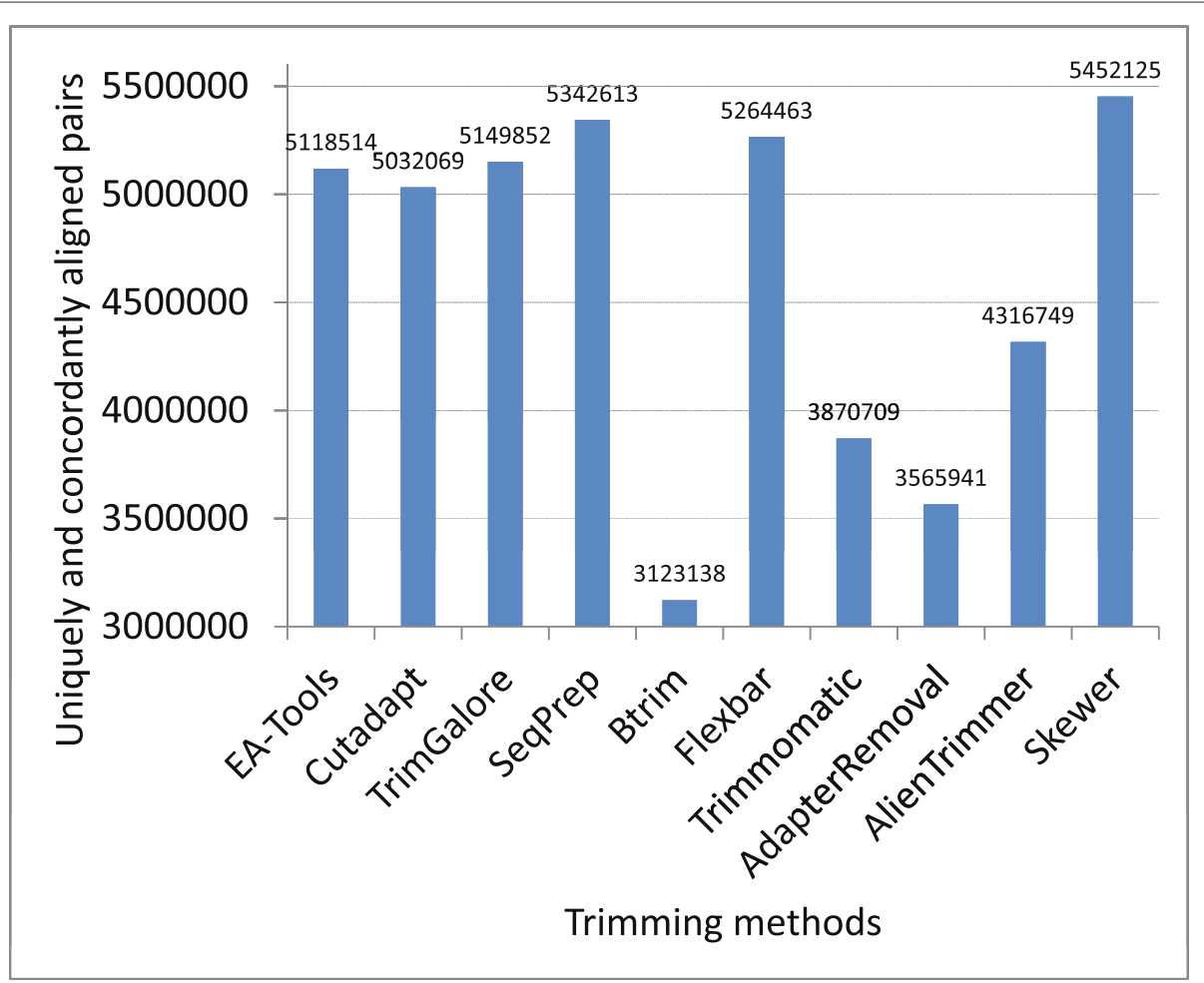

Figure 4 Performance of various adapter trimmers on real paired-end data [SRA:SRR330569].

The time needed for the adapter trimming and the N50 lengths of the scaffolds were used as the metrics for the evaluation.

The result is listed in Table 3 (see Additional file 4 for relevant commands in detail), from which we can see that Skewer marginally outperforms NextClip in terms of assembly statistics (N50 length etc.) of the trimmed reads. In addition, Skewer is about $49 \%$ faster than NextClip in single thread mode.

\section{Conclusions}

We presented a novel algorithm and applied it to adapter trimming. The inherent advantage of the proposed bitmasked $k$-difference matching dynamic programming algorithm makes it possible to search adapter sequence pattern in an exhaustive yet efficient manner. Moreover, by using carefully designed scoring schemes for adapter pattern matching in both SE and PE sequencing data, the resultant Skewer tool was shown to achieve accuracies that were not matched by other similar tools that are currently available. Importantly, Skewer was not optimized for specific applications (e.g. sRNA sequencing); however, compared with other adapter trimmers, it performed well over all NGS applications.

Read lengths and throughputs of NGS technologies are likely to keep increasing; therefore, efficient and accurate adapter trimming methods will continue to be important in the preprocessing steps in applications such as genome resequencing, de novo sequencing, transcriptome sequencing, as well as sRNA sequencing.

Table 3 Comparison of NextClip and Skewer in processing Nextera long mate-pair (LMP) reads (ERA264981)

\begin{tabular}{|c|c|c|c|c|}
\hline \multirow{2}{*}{ Reads for scaffolding } & \multirow{2}{*}{ Time for trimming adapters from Nextera LMP library (s) } & \multicolumn{3}{|c|}{ Assembly } \\
\hline & & Pieces & N50 length & Total length (Mbp) \\
\hline None & N/A & 14097 & 19150 & 105.80 \\
\hline Paired end (PE) only & N/A & 11781 & 23496 & 104.84 \\
\hline PE and NextClip processed & 1480.39 & 6080 & 309342 & 111.55 \\
\hline \multirow{2}{*}{ PE and Skewer processed } & 993.49 (single thread) & \multirow{2}{*}{5806} & \multirow{2}{*}{312317} & \multirow{2}{*}{112.52} \\
\hline & 155.96 (8 threads) & & & \\
\hline
\end{tabular}




\section{Methods}

\section{Problem definition}

\section{Notation}

The Levenshtein distance between two strings $A=a_{1} a_{2} \ldots$ $a_{|A|}, B=b_{1} b_{2} \ldots b_{|B|}$, noted as $\|A, B\|_{l e v}$, is given by a recursive formula (assuming $|A|>0,|B|>0$ ):

$\|A, B\|_{l e v}:=\min \left\{\begin{array}{l}\left\|a_{1} a_{2} \ldots a_{|A|-1}, b_{1} b_{2} \ldots b_{|B|-1}\right\|_{l e v}+\delta_{a_{|A|}, b_{|B|}} \\ \left\|a_{1} a_{2} \ldots a_{|A|-1}, B\right\|_{l e v}+1 \\ \left\|A, b_{1} b_{2} \ldots b_{|B|-1}\right\|_{l e v}+1\end{array}\right.$

where $\delta_{a, b}=1$ if $a \neq b$, otherwise $\delta_{a, b}=0$. When $\min (|A|,|B|)=0,\|A, B\|_{\text {lev }}:=\max (|A|,|B|)$.

\section{k-difference problem}

Given a sequence $S=s_{1} s_{2} \ldots s_{n}$, a query pattern $P=$ $p_{1} p_{2} \ldots p_{m}$, and a threshold $k(0 \leq k<m)$, search all substrings of $S$, noted as $\left\{P^{\prime}\right\}$, such that $\left\|P^{\prime}, P\right\|_{\text {lev }} \leq k$.

\section{Extended $k$-difference problem}

Given a sequence $S=s_{1} s_{2} \ldots s_{n}$, a query pattern $P=$ $p_{1} p_{2} \ldots p_{m}$, and a threshold $e(0 \leq e<1,\lfloor n \times e\rfloor=$ $k)$, search all substrings of $S$, noted as $\left\{P^{\prime}\right\}$, such that $\left\|P^{\prime}, P\right\|_{l e v} \leq k$; and all suffixes of $S$, noted as $\left\{S^{\prime}\right\}$, such that $\exists$ a prefix of $P$, noted as $P^{\prime}$, and $\left\|S^{\prime}, P^{\prime}\right\|_{\text {lev }} \leq\left|S^{\prime}\right| \times e$

\section{Algorithms}

For the $k$-difference problem, the classic approach [2] computes a $(m+1) \times(n+1)$ dynamic programming matrix $\mathrm{C}[0 . . \mathrm{m}, 0 . . \mathrm{n}]$ using the following recurrence:

$$
C[i, j]=\min \left\{\begin{array}{l}
C[i-1, j-1]+\delta_{i j} \\
C[i-1, j]+1 \\
C[i, j-1]+1
\end{array}\right.
$$

where

$$
\delta_{i j}=\left\{\begin{array}{l}
0, \text { if } p_{i}=s_{j}, \\
1, \text { otherwise }
\end{array}\right.
$$

with initialization at the upper boundary by $C[0, j]=0$, and at the left boundary by $C[i, 0]=i$, for $i=1,2, \ldots, m$ and $j=1,2, \ldots, n$. Finally, the algorithm tests the last row of the matrix, i.e. $C[m, j]$, and reports those elements that are no greater than $k$. This algorithm has $O(m n)$ time and $O(m n)$ space complexity.

The space bound can be easily reduced to $O(m)$ if matrix $C$ is computed by columns, noted as $C_{j}$ for $j=1,2, \ldots n$, and report a match each time $C_{j}[m] \leq k$, because computing column $C_{j}$ requires only the knowledge of previous column $C_{j-1}$. With careful design, $C_{j}$ and $C_{j-1}$ can share one column vector, as proposed by Ukkonen [4].

Ukkonen also observed that for columns that have the last element greater than $k$, there is a boundary index of $C_{j}$, noted as $\operatorname{lac}\left(C_{j}\right)$, such that $C_{j}\left[\operatorname{lac}\left(C_{j}\right)\right]=k$ and $C_{j}[l]>k$ for $l=\operatorname{lac}\left(C_{j}\right)+1, \ldots m$. It is easy to prove that $\operatorname{lac}\left(C_{j}\right) \leq$ lac $\left(C_{j-1}\right)+1$. Using this observation, Ukkonen reduced the time from $O(m n)$ to expected $O(k n)$ [4].

Our algorithm was developed from Ukkonen's algorithm; however, we use a queue instead of an array to store all elements of current column above the boundary index. When there is a new element that corresponds to the topmost element of the new column, all elements in the queue shift automatically to the next (lower) position, just as elements transfer in the diagonals of matrix $C$. This process inherently keeps the basic properties of Ukkonen's algorithm and facilitates subsequent improvements.

Lemma 1. In the dynamic programming matrix $C$ for tackling the $k$-difference problem, the values of elements along each diagonal are monotonically non-decreasing.

The proof is provided in Additional file 5 Appendix A.

Theorem 1. All the matched elements of the query pattern and sequence are equal to their precursors in the diagonal and do not need to be updated in the dynamic programming process.

Proof. This theorem is a direct consequence of Lemma 1 and the dynamic programming recurrence, when $\delta_{i j}=0$.

In other words, only mismatched elements need to be updated in the dynamic programming process. If bit-vectors that denote mismatched positions of comparison between the adapter sequence and each of the four nucleotide characters are pre-computed and a bitvector that marks all positions of the queue elements that exceed the $k$-difference constraint is maintained, then unnecessary computations in updating the column vector can be inhibited. This is the key point that led to the main improvement of our algorithm over Ukkonen's algorithm.

As listed in Algorithm 1, the bit-masked $k$-difference matching algorithm has the following characteristics:

- Use a queue instead of an array to store all elements of the current column above boundary index.

- In preprocessing, calculate for each of four nucleotide characters a bit-vector that denotes the mismatched positions compared with the adapter.

- Mark the internal cells that exceed the $k$-difference constraint by a bit-vector which shifts as the queue pushes it.

- When processing the column starting from each input nucleotide, update only the cells that mismatch and have not been marked.

This algorithm uses a queue of size $m$ and several bit vectors of size $\lceil m / w\rceil$, where $w$ is the word length of the computer (for example $w$ equals 64 for a 64-bit machine), 
and hence has a space of $O(m)$. For each of the $n$ characters in a target sequence, the character enters the queue once and exits from the queue at most once. For a random sequence, the expected size of the queue is $O(k)$; hence, generally the algorithm has $O(k n)$ expected time. However, because it is restricted by the bit-mask operations, each element in the queue usually updates at most $k+1$ times. Because bit operations are negligible compared with element update operations, this algorithm achieves $O(k n)$ worst-case time in practice, which is better than the $O(k n)$ expected time for Ukkonen's algorithm.

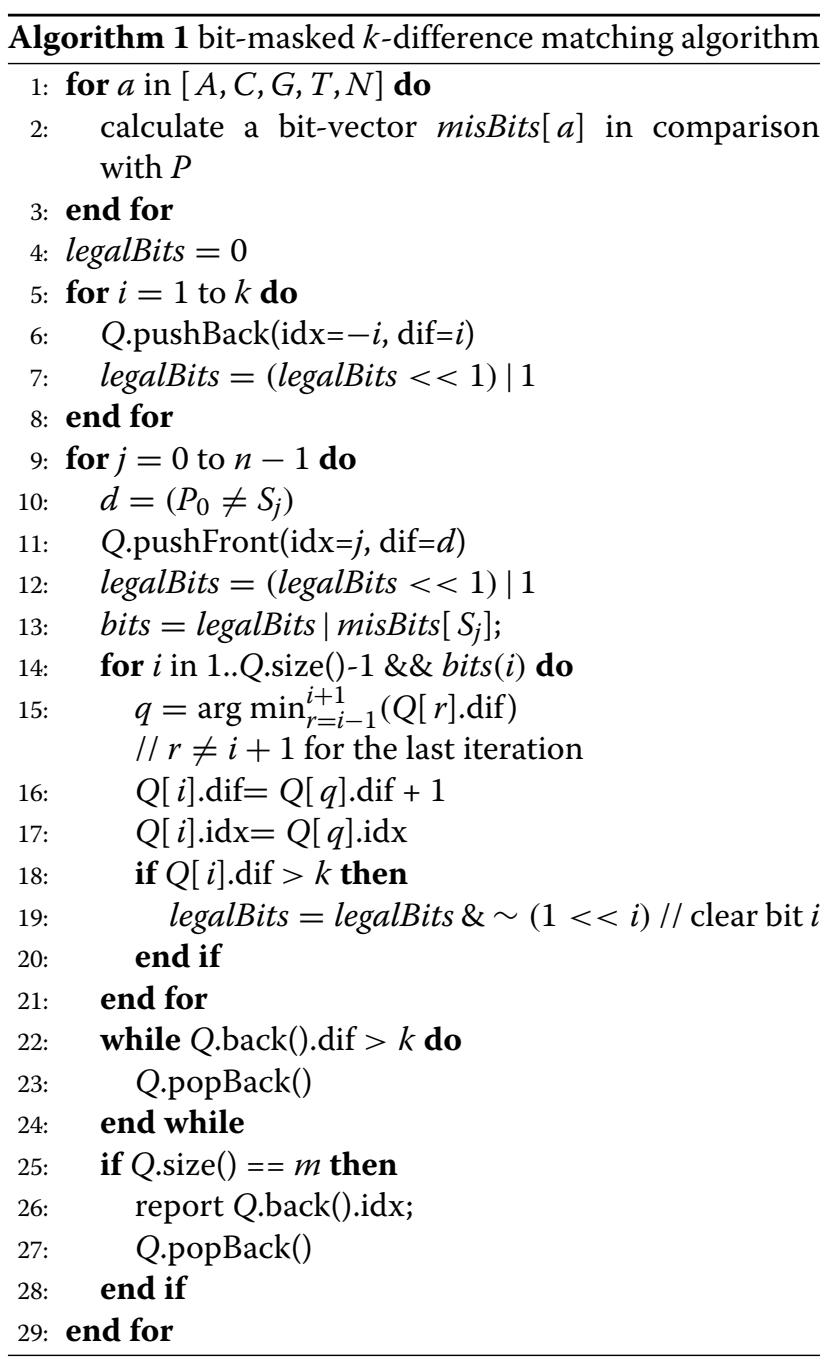

Algorithm 1 can be improved further by avoiding all unnecessary updates through constant time bit operations within each iteration cycle of the target nucleotide. The basic principle is that when an element in a diagonal of the original dynamic programming matrix has a value that is derived from an adjacent diagonal (i.e. an indel occurs in the corresponding path), the score and associated index of the element will remain unchanged if the precursor remains unchanged.

Although the above improvement can reduce the theoretical time complexity from expected $O(k n)$ to worst-case $O(k n)$, experiments on large volumes of real data showed that the reduced element update operations did not compensate for the additional bit operations.

For the extended $k$-difference problem, an additional step bounded by $O(m)$ is performed to check all the elements remained in the queue if no hit was found in previous steps.

\section{Deal with base-call qualities}

The main advantage of the bit-masked k-difference matching algorithm over Myer's bit-vector algorithm is that it can be extended to handle base-call quality values.

To handle base-call quality values, we introduce the following parameters: $P_{\min }=-\log _{10}(1 / 3)$, the minimum penalty for a mismatch; $P_{\max }=-\log _{10}\left(10^{40 /(-10)} / 3\right)$, the maximum penalty for a mismatch; delta $=P_{\max }$, the penalty for an insertion or deletion. The penalty of a mismatch with quality value $q$ is calculated as:

$$
P(q)=\left\{\begin{array}{cc}
P_{\min } & q \leq 0 \\
P_{\text {min }}+q / 40 \times\left(P_{\max }-P_{\text {min }}\right) & 0<q<40 \\
P_{\max } & q \geq 40
\end{array}\right.
$$

It is trivial to prove that $P(q)=-\log _{10}\left(10^{q /(-10)} / 3\right)$ when $0<q<40$. This score is the negative logarithm of the probability that the corresponding base is actually a match to the adapter sequence with sequencing error.

Note that the scoring scheme herein only induces penalties for mismatches and insertions/deletions. For matches, because the possibility of false matching based on sequence homology reduces exponentially as the matching length increases, the false matching possibility can be reduced by setting a longer alignment length.

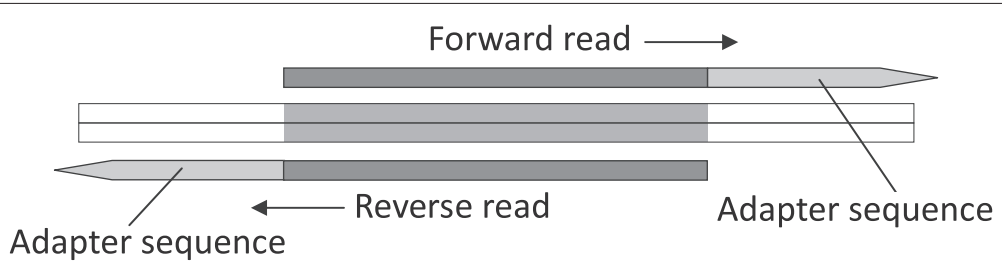

Figure $\mathbf{5}$ Layout of paired-end reads that have adapter contaminants. 
When PE information is available, the false matching possibility can be reduced to a minimum.

The extended version of Algorithm 1 that can take quality values into consideration is outlined in Additional file 6 Appendix B.

\section{Deal with paired-end information}

Unlike the standard SE sequencing, PE sequencing reads out each DNA template twice, in opposite directions from different ends. The underlying fact is that all the $P E$ reads that need to be trimmed must have the preserved paired sequences reverse-complement to each other, as illustrated in Figure 5 and Additional file 7 Appendix C.

Using this property, the program first finds all $k$ difference occurrences of adapters in both paired reads using the extended version of Algorithm 1 with quality values considered. Then the reverse-complementary property of each trimmed paired sequences is checked. Next, all candidates are evaluated with a scoring scheme that takes into account the fitness of adapter sequences in paired reads and the alignment of reverse-complementary counter-parts. Finally, the program outputs the optimal occurrence, if any.

The scoring scheme we used is as follows:

$$
\begin{aligned}
\operatorname{score}(i d x)= & p s c o r e(\operatorname{read} 1[i d x \ldots \operatorname{readLen}-1], \text { adapter } 1) \\
& +p s c o r e(\operatorname{read} 2[i d x \ldots \operatorname{readLen}-1], \text { adapter } 2) \\
& +\operatorname{pscore}(\operatorname{read} 1[0 \ldots i d x-1] \\
& \operatorname{rev} \operatorname{Comp}(\operatorname{read} 2[0 \ldots i d x-1]))
\end{aligned}
$$

where $i d x$ is the start position for trimming, $\operatorname{pscore}(x, y)=$ $|x| \times P_{\max }-$ penalty $(x, y), P_{\max }$ is the maximum penalty for a difference, penalty $(x, y)$ is the penalty for matching $x$ and $y$ as calculated by the $k$-difference matching algorithms, and $\operatorname{revComp}(x)$ denotes the reverse complementary sequence of $x$. The goal is to find the $i d x$ that meets the $k$-difference requirement and maximizes the score function.

\section{Deal with Nextera LMP information}

Mate-pair library sequencing allows the generation of long-insert PE libraries that are useful in the scaffolding process of de novo genome assembly and in the detection of long-range genome structural variations. In the Nextera LMP library construction process, there are additional reactions called "tagmentation" and "circularization" before the normal PE library construction. The tagmentation reaction uses a specially engineered transposome to fragment the DNA sample and tag the DNA fragments by attaching a pair of biotinylated junction adapters simultaneously to the ends. Next, the tagmented DNA molecules are circularized and sheared by ultrasonics, and the sub-fragments containing the original junction parts are enriched via the biotin tag in the junction adapter.

Trimming adapters from Nextera LMP reads is like a reverse process of Nextera LMP library construction. To process Nextera mate-pair reads, the program first trims the adapters as if it is dealing with PE reads. Then, it trims junction adapters from the processed paired reads separately using the extended version of Algorithm 1.

\section{Availability of supporting data}

The data sets supporting the results of this article are available from high-throughput DNA and RNA sequence read archive: http://www.ncbi.nlm.nih.gov/sra/?term=SRR014966, http://www.ncbi.nlm.nih.gov/sra/?term=SRR330569, http:// www.ncbi.nlm.nih.gov/sra/?term=ERA264981, and http:// www.ncbi.nlm.nih.gov/sra/?term=SRR519624.

\section{Additional files}

Additional file 1: Tabular metrics of experiments on simulated data. Additional file 2: Tabular metrics of experiments on real sRNA data. Additional file 3: Tabular metrics of experiments on real PE RNA-Seq data.

Additional file 4: Commands for conducting experiments on real LMP data.

Additional file 5: Proofs of some fundamental lemmas.

Additional file 6: Details of extended algorithms.

Additional file 7: An example for adapter trimming of real PE data.

\section{Competing interests}

The authors declare that they have no competing interests.

\section{Authors' contributions}

SZ and SWD supervised the project. HJ conceived the algorithm, developed the program, and performed the data analysis. RL explained NGS protocols and provided biological perspectives on trimming effects. All authors read and approved the final manuscript.

\section{Acknowledgements}

We thank Dr. Zhongxin Guo for providing a large volume of the real genome resequencing data and sRNA sequencing data used for this study. This work was supported by the National Key Technology R\&D Program of China (2012BAK11B01), the Basic Scientific Research Foundation of the Chinese Academy of Inspection and Quarantine (CAIQ) (2013JK003), and the Special Fund of CAIQ for Pest Identification and Monitoring.

\section{Author details}

${ }^{1}$ Institute of Plant Quarantine Research, Chinese Academy of Inspection and Quarantine, Huixinli 241, Beijing, 100029 China. ${ }^{2}$ Department of Plant Pathology and Microbiology and Institute for Integrative Biology, University of California, Riverside, 900 University Ave, 92521 Riverside, USA. 


\section{References}

1. He HH, Meyer CA, Hu SS, Chen MW, Zang C, Liu Y, Rao PK, Fei T, Xu H, Long H, Liu XS, Brown M: Refined dnase-seq protocol and data analysis reveals intrinsic bias in transcription factor footprint identification. Nature Methods 2014, 11(1):73-78.

2. Smith TF, Waterman MS: Identification of common molecular subsequences. J Mol Biol 1981, 147(1):195-197.

3. Martin M: Cutadapt removes adapter sequences from high-throughput sequencing reads. EMBnet.journal 2011, 17:10-12.

4. Ukkonen E: Finding approximate patterns in strings. J Algorithm 1985, 6(1):132-137.

5. Myers $\mathrm{G}$ : $\mathbf{A}$ fast bit-vector algorithm for approximate string matching based on dynamic programming. J ACM 1999, 46(3):395-415.

6. Kong $Y$ : Btrim: a fast, lightweight adapter and quality trimming program for next-generation sequencing technologies. Genomics 2011, 98(2):152-153.

7. Falgueras J, Lara AJ, Fernandez-Pozo N, Canton FR, Perez-Trabado G, Claros MG: Seqtrim: a high-throughput pipeline for pre-processing any type of sequence read. BMC Bioinformatics 2010, 11:38.

8. Schmieder R, Lim YW, Rohwer F, Edwards R: Tagcleaner: Identification and removal of tag sequences from genomic and metagenomic datasets. BMC Bioinformatics 2010, 11:341.

9. Dodt M, Roehr JT, Ahmed R, Dieterich C: Flexbar-flexible barcode and adapter processing for next-generation sequencing platforms. Biology (Basel) 2012, 1(3):895-905.

10. Needleman SB, Wunsch CD: A general method applicable to the search for similarities in the amino acid sequence of two proteins. J Mol Biol 1970, 48(3):443-453.

11. Lohse M, Bolger AM, Nagel A, Fernie AR, Lunn JE, Stitt M, Usadel B: Robina: a user-friendly, integrated software solution for rna-seq-based transcriptomics. Nucleic Acids Res 2012, 40(Web Server issue):622-627.

12. Lindgreen S: Adapterremoval: easy cleaning of next-generation sequencing reads. BMC Res Notes 2012, 5:337.

13. Criscuolo A, Brisse S: Alientrimmer: a tool to quickly and accurately trim off multiple short contaminant sequences from high-throughput sequencing reads. Genomics 2013, 102(5-6):500-506.

14. Leggett RM, Clavijo BJ, Clissold L, Clark MD, Caccamo M: Nextclip: an analysis and read preparation tool for nextera long mate pair libraries. Bioinformatics 2014, 30(4):566-568,

15. Huang W, Li L, Myers JR, Marth GT: Art: a next-generation sequencing read simulator. Bioinformatics 2012, 28(4):593-594.

16. Kato M, de Lencastre A, Pincus Z, Slack FJ: Dynamic expression of small non-coding rnas, including novel micrornas and pirnas/21u-rnas, during caenorhabditis elegans development. Genome Biol 2009, 10(5):54.

17. Hillier LW, Marth GT, Quinlan AR, Dooling D, Fewell G, Barnett D, Fox P, Glasscock JI, Hickenbotham M, Huang W, Magrini VJ, Richt RJ, Sander SN, Stewart DA, Stromberg M, Tsung EF, Wylie T, Schedl T, Wilson RK, Mardis ER: Whole-genome sequencing and variant discovery in c. elegans. Nat Methods 2008, 5(2):183-188.

18. Langmead B, Salzberg SL: Fast gapped-read alignment with bowtie 2. Nat Methods 2012, 9(4):357-359.

19. Trapnell C, Pachter L, Salzberg SL: Tophat: discovering splice junctions with rna-seq. Bioinformatics 2009, 25(9):1105-1111.

20. Kim D, Pertea G, Trapnell C, Pimentel H, Kelley R, Salzberg SL: Tophat2: accurate alignment of transcriptomes in the presence of insertions, deletions and gene fusions. Genome Biol 2013, 14(4):36.

21. Consortium $\mathrm{F}$ : The flybase database of the drosophila genome projects and community literature. Nucleic Acids Res 2003, 31(1):172-175.

22. Del Fabbro C, Scalabrin S, Morgante M, Giorgi FM: An extensive evaluation of read trimming effects on illumina ngs data analysis. PLoS One 2013, 8(12):85024.

23. Simpson JT, Wong K, Jackman SD, Schein JE, Jones SJ, Birol I: Abyss: a parallel assembler for short read sequence data. Genome Res 2009, 19(6):1117-1123.

doi:10.1186/1471-2105-15-182

Cite this article as: Jiang et al:: Skewer: a fast and accurate adapter trimmer for next-generation sequencing paired-end reads. $B M C$

Bioinformatics 2014 15:182.

Submit your next manuscript to BioMed Central and take full advantage of:

- Convenient online submission

- Thorough peer review

- No space constraints or color figure charges

- Immediate publication on acceptance

- Inclusion in PubMed, CAS, Scopus and Google Scholar

- Research which is freely available for redistribution

Submit your manuscript at www.biomedcentral.com/submit
C Biomed Central 